Article

\title{
Frequency Dependence of Electrical Parameters of an Organic-Inorganic Hybrid Composite Based Humidity Sensor
}

\author{
Rizwan Akram \\ Department of Electrical Engineering, College of Engineering, Qassim University, P.O. Box 6677, \\ Qassim 51452, Saudi Arabia; rizwanakram75@qec.edu.sa; Tel.:+966(538)264603 \\ Academic Editor: Thomas Riedl \\ Received: 11 February 2016; Accepted: 12 May 2016; Published: 23 May 2016
}

\begin{abstract}
The present study highlights the interdependence of ambient humidity levels on the electrical parameters of organic-inorganic hybrid composite based humidity sensor at varied AC frequencies of input signal. Starting from the bottom, the layer stack of the fabricated humidity sensor was 200-nm silver (Ag) thin film and $4 \mu \mathrm{m}$ spun-coated $\mathrm{PEPC}+\mathrm{NiPC}+\mathrm{Cu}_{2} \mathrm{O}$ active layer. Silver thin films were deposited by thermal evaporator on well cleaned microscopic glass slides, which served as a substrate. Conventional optical lithography procedure was adapted to define pairs of silver-silver surface electrodes with two sorts of configurations, i.e., interdigitated and rectangular. Humidity-sensitive layers of organic-inorganic composite were then spun-cast upon the channel between the silver electrodes. The changes in relative humidity levels induced variation in capacitance and impedance of the sensors. These variations in electrical parameters of sensors were also found to be highly dependent upon frequency of input AC signal. Our findings reveal that the organic-inorganic composite shows higher humidity sensitivity at smaller orders of frequency. This finding is in accordance with the established fact that organic semiconductors-based devices are not applicable for high frequency applications due to their lower charge carrier mobility values. Two distinct geometries of semiconducting medium between the silver electrodes were investigated to optimize the sensing parameters of the humidity sensor. Furthermore, the effect of temperature change on the resistance of organic composite has also been studied.
\end{abstract}

Keywords: organic-inorganic hybrid composite; photolithography; dielectric medium; polarizability; multi frequency impedance spectrum

\section{Introduction}

The need for sensors in the environmental monitoring and medical diagnostics domain is ever increasing [1-4]. For instance, in neonatal pediatrics, survival of newborn baby is highly dependent on the incubator's relative humidity level [5]. The humidity control is also necessary in moisture-sensitive environments, such as in pharmaceutical industries, food storages, building air-conditioning, museums, paper industry and libraries. Resultantly, in view of their significant technological importance, there is rapid and continued advancement in fabrication of economical, miniaturized and sensitive humidity sensors $[6,7]$.

So far, several materials have been opted for humidity sensing application. The most notable ones include porous silicon, ceramics, $\mathrm{LiCl}$ and silica aerogel [8-11]. Each of them has its own merits and shortcomings; however, the operational mechanism of most of these sensors is the protonic conducting mechanism of the Grotthuss type [12]. Due to condensation of water molecules, the aquatic layer gets stacked up on the sensing material surface, allowing protons to migrate freely throughout the surface of the sensor. Thus, the change in ambient humidity level is detected by the 
increment in conductivity of the sensing layer. However, despite the robustness, stability and high sensitivity of these inorganic based humidity sensors, there are a couple of compelling reasons which motivate researchers to focus their efforts towards organic semiconductors [13]. In contrast to inorganic semiconductors, organic materials are environmentally and economically favorable [14-16]. Further, by utilizing solution processable organic materials, the tedious vacuum deposition techniques can be replaced by unsophisticated fabrication procedures [17-20].

Several organic materials including cellulose, acetate butyrate, methyl red, phthalocyanine and polyimide have also been studied for humidity sensing application [21-28]. The mode of operation for organic based sensors is either capacitive type or resistive type sensing. However, despite the advantages, viz., low power consumption and linearity in response, mostly humidity sensors are based on capacitive type sensing. Their working principle is based upon the dielectric permittivity variation resulting from absorption of water vapors by a sensing layer. In recent years, in addition to organic materials, metal oxide semiconductors (MOS) like aluminum oxide $\left(\mathrm{Al}_{2} \mathrm{O}_{3}\right)$, copper oxide $\left(\mathrm{Cu}_{2} \mathrm{O}\right)$, iron oxide $\left(\mathrm{Fe}_{2} \mathrm{O}_{3}\right)$, Yttrium oxide $\left(\mathrm{Y}_{2} \mathrm{O}_{3}\right)$ and tungsten trioxide $\left(\mathrm{WO}_{3}\right)$ have emerged as another economical alternative for fabrication of humidity sensors [29]. To incorporate the advantages of metal oxides, some researchers have also used organic-inorganic composite to sense ambient humidity [30,31]. Resultantly, researchers combine the advantages of both organic and inorganic materials with the possibility to utilize existing solution processable semiconductor fabrication procedures.

Earlier we studied the humidity sensing capability of a blend of copper oxide $\left(\mathrm{Cu}_{2} \mathrm{O}\right)$ and poly-N-epoxy propyl carbazole (PEPC), by virtue of porosity in its structure [32]. Later, to enhance the sensitivity of the sensor we added an organic material from the phthalocyanine family: Nickel phthalocyanine ( $\mathrm{NiPc}$ ), and investigated the organic-inorganic composite of $\mathrm{PEPC}, \mathrm{NiPC}$ and $\mathrm{Cu}_{2} \mathrm{O}$ as humidity sensor [33]. It is well known that hydrophobic materials are generally used for fabrication of humidity sensors, otherwise the sensing layer may peel off due to dissolution in water at higher humidity levels. Because it is insoluble in water, we considered the organic-inorganic hybrid composite $\left(\mathrm{Cu}_{2} \mathrm{O}-\mathrm{PEPC}-\mathrm{NiPC}\right)$ an ideal candidate for humidity monitoring. In our previous study, the morphological study and compositional analysis of the composite film has already been dealt with in detail. However, so far, in the aforementioned studies, capacitive response in a wide frequency range $(300 \mathrm{~Hz}-100 \mathrm{KHz})$ has been neglected. The multi frequency impedance spectrum of the $\mathrm{Cu}_{2} \mathrm{O}-\mathrm{PEPC}-\mathrm{NiPC}$ based humidity sensor has also not been investigated.

The present work aims to highlight the effect of humidity on capacitance along with the impedance of $\mathrm{Ag} / \mathrm{Cu}_{2} \mathrm{O}-\mathrm{PEPC}-\mathrm{NiPC} / \mathrm{Ag}$ surface type humidity sensor. The interdependence of electrical parameters (capacitance and impedance) with frequency has been analyzed in detail, which helps us understand both the basic electrical properties of humidity sensor and operational mechanism of the humidity sensor. The sensing parameters of the sensors are believed to be dependent upon the choice of the hygroscopic material as well as electrode geometry. Hence, we have compared the effect of electrode configuration for the optimization of the sensor's response as well.

\section{Experimental}

The precursor solution for the hybrid organic-inorganic humidity sensing layer was primarily prepared by adding $7 \mathrm{wt} \%$ Copper oxide, $4 \mathrm{wt} \%$ poly epoxy propyl carbazole and $5 \mathrm{wt} \%$ nickel pthalocyanine in benzol (Sigma Aldrich, Lahore, Pakistan). For our study, PEPC (Mw: 1200 units) was synthesized in the laboratory whereas $\mathrm{Cu}_{2} \mathrm{O}$ micro-particles (diameter $\sim 3-4 \mu \mathrm{m}$ ) and Nickel Phthalocyanine were purchased from WINLAB UK (Market Harborough, UK) and Sigma Aldrich (Lahore, Pakistan) respectively.

Commercially available microscopic glass slides were well cleaned following the well-established protocol. This involved rinsing glass slides in Deionized (DI) water (SILEX ${ }^{\mathrm{TM}}$ deionizer, Dayton, $\mathrm{OH}, \mathrm{USA}$ ), acetone (Sigma Aldrich, Lahore, Pakistan), ethanol (Sigma Aldrich, Lahore, Pakistan) and finally again in DI water for 5 min each. To dry the glass substrates, nitrogen gas (Fine gas company, Lahore, Pakistan) was blown on them in a dust free environment. Edward Auto 306 thermal 
evaporator (Edwards High Vacuum, Sussex, UK) was later used to deposit thin film of silver on the glass substrates. A film thickness of $200 \mathrm{~nm}$ was obtained by passing $22 \mathrm{~A}$ current through the tungsten filament for $\sim 2 \mathrm{~m}$. Meanwhile the pressure inside the thermal evaporator was kept below $10^{-5}$ mbar. Silver thin film was then subjected to optical lithography so as to pattern it into a pair of electrodes. The standard lithography procedure was adopted to define the pair of electrodes with two distinct configurations, i.e., rectangular and interdigitated. Figure 1 summarizes the whole lithographic for patterning the silver film into a pair of rectangular electrodes separated by a gap of $\sim 20 \mu \mathrm{m}$. The same procedure was adapted to pattern the silver thin film into a pair of interdigitated electrodes.

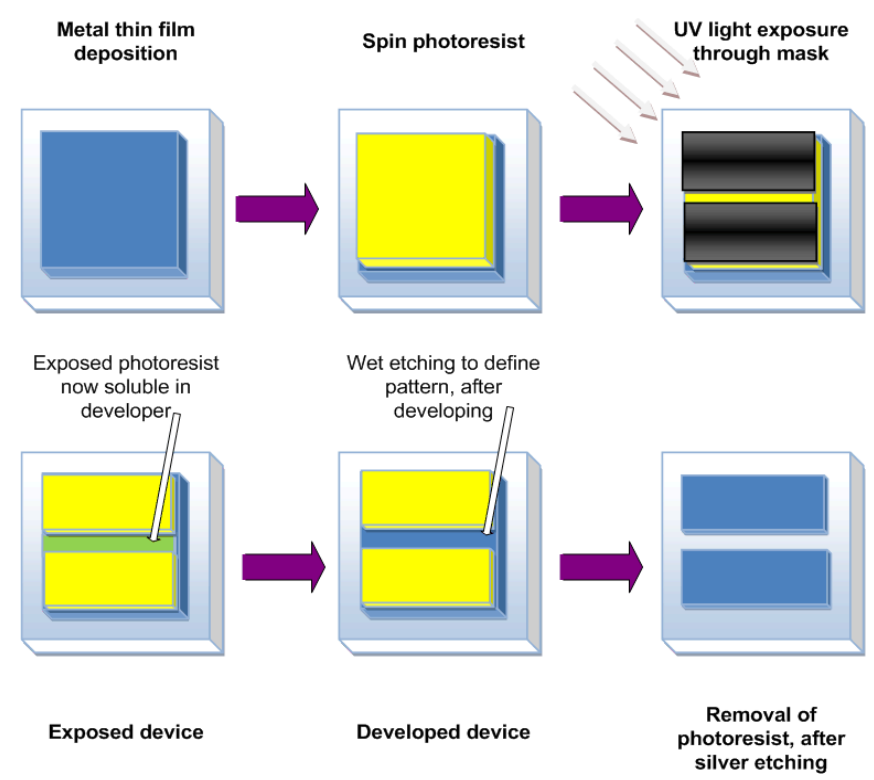

Figure 1. Photolithography procedure to define pair of rectangular electrodes on glass substrate.

The hygroscopic property of the material is considered vital for humidity sensing and it depends upon the porosity and pore geometry of the sensing material as well as electrode geometry. The interdigitated electrodes configuration allows the water vapors to diffuse freely into the dielectric. Hence, our study involves the comparison of rectangular and interdigitated electrodes for the optimization of the humidity sensor.

Finally, to complete the fabrication process, organic-inorganic hybrid blended film was spun cast upon the channel between the electrodes. Spin-coater P-6000 (Itegrated technologies, Sussex, UK) with angular velocity of 2000 RPM was used to produce film thicknesses of $4 \mu \mathrm{m}$. This humidity sensing film covered the gap between the silver electrodes to later function as dielectric medium. The schematic structure of the humidity sensor and the top view of the electrodes geometries are depicted in Figure 2.

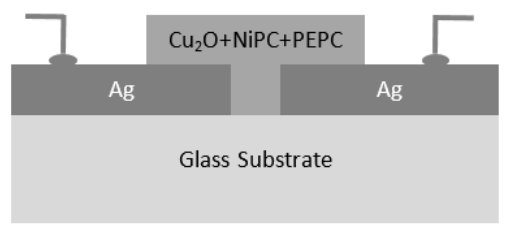

(a)

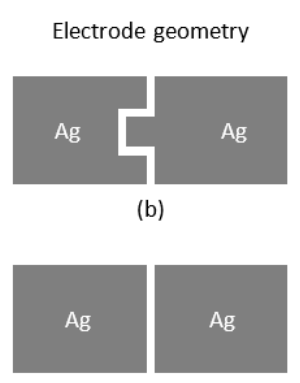

(c)

Figure 2. (a) Cross-sectional view of organic-inorganic hybrid composite based humidity sensor; top view of (b) interdigitated and (c) rectangular electrodes configuration. 
The humidity sensing property of the sensors was studied by investigating their electrical response as a function of altering relative humidity $(\mathrm{RH})$ levels. The humidity sensors were placed in a home-made sealed chamber, wherein humidity level was varied by influx of dry and wet nitrogen gas. To increase the humidity level in the chamber, nitrogen gas was primarily passed through water and then introduced into the humidity chamber. The influx of wet nitrogen gas (N2 gas loaded with moisture) helped in increasing the RH levels inside the sealed chamber. The use of sealed chamber helped in maintaining the higher RH at a constant level inside the chamber. The same approach, to vary the RH level inside the chamber, has been previously utilized in various studies [34,35]. Capacitance and real and imaginary impedance components of the sensors were then recorded as a function of varied humidity levels. SR 830 lock-in amplifier from stanford research instruments (Sunnyvale, California, USA) in amplifier with applied AC voltage of $1 \mathrm{~V}$ and the frequency ranging from $1 \mathrm{~Hz}$ to $100 \mathrm{kHz}$ was utilized for capacitance and impedance measurements. Humidity sensors were calibrated by a commercially available hygrometer "Mastech MS 6503" (Dongguan Huayi Mastech Company Limited, Dongguan, China), with measurement accuracy of $\pm 1 \% \mathrm{RH}$ at $25^{\circ} \mathrm{C}$.

\section{Results and Discussion}

Bode plots of the humidity sensors were obtained by recording the capacitance of the sensors as a function of humidity at room temperature. Figure $3 \mathrm{a}, \mathrm{b}$ depicts the capacitance variation of the sensors with interdigitated and rectangular (Ag) electrodes geometry, respectively.

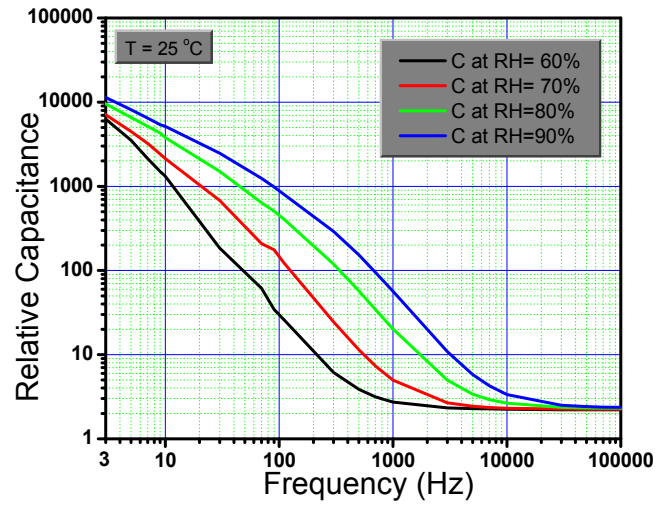

(a)

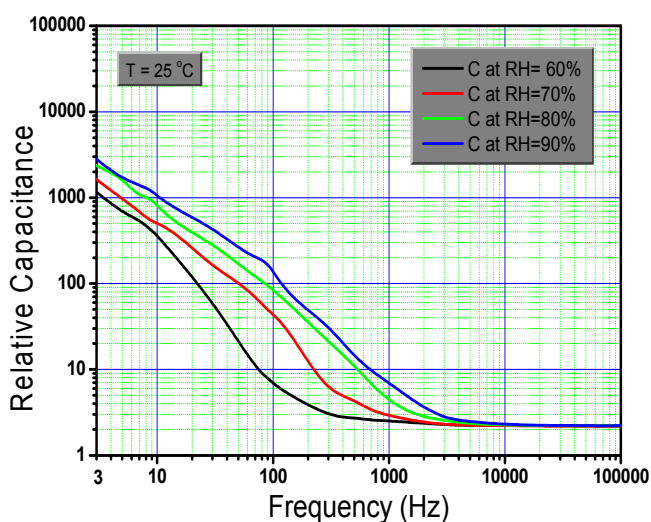

(b)

Figure 3. The capacitance variation of the sensors (as a function of humidity at varied frequencies) with (a) interdigitated and (b) rectangular (Ag) electrodes configuration respectively.

For both sensors, increase in the capacitance is observed with the increment in the relative humidity (RH) of the surrounding air. It is inferred from the experimental results that the sensor with interdigitated electrode configuration shows higher humidity sensitivity as compared to the counter one. The capacitance of the sensors is basically dependent upon several parameters, i.e., the area $(A)$ and the separation between the parallel plates $(d)$ and polarizability of the dielectric medium present in between the plates. In the case of our humidity sensor, the organic-inorganic hybrid blend serves the purpose of dielectric and its polarizability $\left(\alpha_{\mathrm{r}}\right)$ itself is of several origins. In organic-inorganic materials hybrid blended thin film, it may have dipolar $\left(\alpha_{\text {dip }}\right)$, ionic $\left(\alpha_{\mathrm{i}}\right)$ and electronic polarizability $\left(\alpha_{\mathrm{e}}\right)$. Hence the Equation (1), [36].

$$
\alpha_{\mathrm{r}}=\alpha_{\mathrm{dip}}+\alpha_{\mathrm{i}}+\alpha_{\mathrm{e}}
$$

In particular, for inorganic ionic compounds like $\mathrm{Cu}_{2} \mathrm{O}$, ionic and dipolar polarization is more frequently observed. With the increase in water contents inside humidity sensing film, dipoles and ions $\left(\mathrm{Cu}^{+}, \mathrm{O}^{2-}, \mathrm{H}^{+}\right.$and $\left.\mathrm{HO}^{-}\right)$formation increases the polarizability of the film. However, for the case of organic materials, relative displacement of the orbital electrons causes electronic polarizability in the 
presence of external electric field. Hence, the enhanced value of capacitance at higher humidity level can be explained in accordance with the Equation (1). With the absorption of water molecules by the organic-inorganic composite film, the dielectric permittivity of the film increases, thereby increasing the value of capacitance of the sensor.

$$
C=\frac{\varepsilon_{o} \varepsilon_{r} A}{d}
$$

Here, $\varepsilon_{\mathrm{o}}$ is the permittivity of space, " $\varepsilon_{\mathrm{r}}$ " is the relative permittivity of the dielectric material between the plates; " $A$ " is the area of the parallel metallic plates and " $d$ " is the separation between them.

The reason for enhanced sensitivity for sensors with interdigitated electrodes is that, as the contact area between the sensing material and the electrodes increases, so does the absorption of water molecules and hence the polarizability of the sensing film. Therefore, high humidity sensitivity is observed for the interdigitated electrode geometry as compared to the rectangular electrode configuration.

Further interesting information that we observe from Figure 3 is the relative change of capacitance as a function of humidity that has been found to be dependent upon frequency of AC signal. At low order frequencies, the increase in capacitance of the sensors is quite sharp with the increase of humidity levels. However, this trend is not observed at higher order of frequencies. Our findings thus reveal that the organic-inorganic composite shows higher humidity sensitivity at low order frequencies of AC signal. In fact, this finding is in accordance with the established fact that organic semiconductors are not suitable for high frequency applications due to their lower charge mobility values. In particular, this trend is pretty common for capacitive type sensors. In fact, after a certain frequency, electric charge carriers present in the sensing film are unable to follow the alteration of applied AC signal. Hence, at higher order of frequency ( $10 \mathrm{~K}$ to $100 \mathrm{~K})$, variation of capacitance with the humidity levels increment is depressed and only a slight variation of capacitance is observed.

As for the capacitance-frequency relationship, we observe a decrease in capacitance with an increase in frequency. Kim et al. [8] explain well the dependency of relative dielectric permittivity of water upon the frequency of AC signal. In fact, at low order frequencies, capacitance of the sensor is determined by the dielectric permittivity of water $\left(\varepsilon_{\mathrm{W}}=80\right)$. However, at higher frequencies, as polarization of water molecules cease to contribute to the polarization of the dielectric; therefore, sensing film's net polarization drops to the permittivity of dry organic-inorganic composite film, which is smaller compared to the permittivity of water. Hence, a decrease in capacitance is observed with increasing frequency of AC signal.

Figure $4 \mathrm{a}, \mathrm{b}$ shows the relationship of the real component of impedance and phase angle with humidity for the humidity sensors with interdigitated and rectangular electrode configuration, respectively. The response was studied with the input signal's frequency ranging from $300 \mathrm{~Hz}$ to 100 $\mathrm{kHz}$. It can be inferred from the experimental data that at low frequency of input signal, the impedance spectra of both sensors exhibits a strong reliance upon the humidity level. The real component of sensor's impedance $\left(Z_{R}\right)$ decreases quite sharply with the increase in humidity level. However, at high frequency, only a slight decrement of $Z_{R}$ is observed with increase in humidity level.

The phase angle of impedance $\left(Z_{\theta}\right.$ calculated in radians) shows an increase with humidity level increment. At higher frequency, there is less variation in phase angle with the increase in humidity. At a particular humidity level, $Z_{\theta}$ shows interesting variation with frequency: initially it shows decreases with frequency increment but after a critical frequency it starts increasing. In fact, below that critical frequency, the capacitance of the device strongly contributes to the phase angle of impedance $\left(Z_{\theta}\right)$. As is known, the capacitance of the device shows a continuous decrease in its value with the frequency increment, so does the $Z_{\theta}$. However, above the critical frequency, the phase angle of impedance $\left(Z_{\theta}\right)$ starts increasing. This could be explained by the fact that at higher frequency, the contribution of capacitance of the device is depressed and the effect of sensor's resistance starts to dominate. The same trend for phase angle of the impedance of the device after a critical frequency has also been observed by other researchers $[37,38]$. Therefore, an increase in value of $Z_{\theta}$ after a critical frequency may be 
attributed to the resistance of the device. Dielectric permittivity and mobility of charge carriers are believed to be responsible for the interdependence of the impedance of the sensor and frequency of the AC signal.

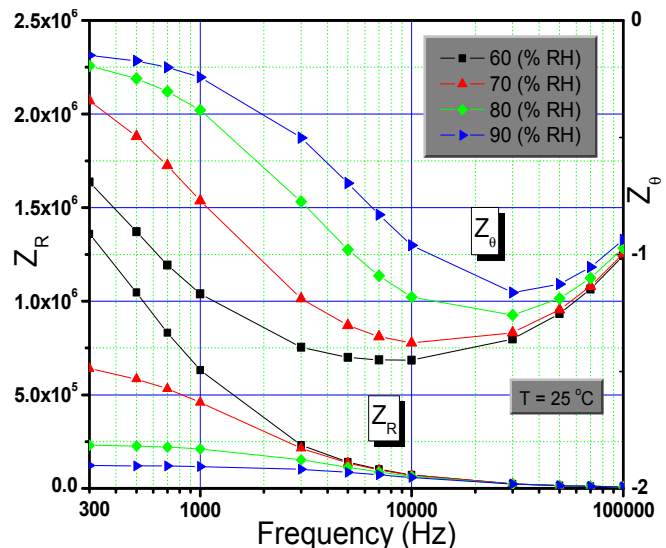

(a)

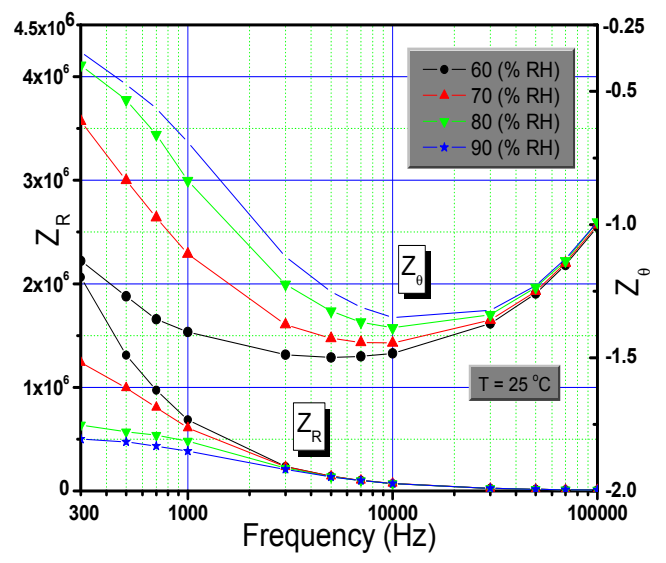

(b)

Figure 4. The variation of real component of impedance and phase angle (as a function of humidity at varied frequencies) of the sensors with (a) interdigitated and (b) rectangular (Ag) electrodes configuration respectively.

It is known that ambient temperature greatly affects the resistance of the materials, and thus it is supposed to affect the impedance of the humidity sensor as well. Figure 5 portrays the resistance of the humidity sensor at various temperatures, when subjected to $60 \% \mathrm{RH}$. It is worth noting that by utilization of sealed chamber, the RH inside the chamber remains unaffected when the sensor is subjected to elevated temperatures (as observed through humidity meter placed inside the chamber). It can be clearly seen from experimental results that the resistance of the organic-inorganic blended film showed a slight decrease (from $538.9 \mathrm{~K} \Omega$ to $465.5 \mathrm{~K} \Omega$ ) in its value with temperature elevation (from $30^{\circ} \mathrm{C}$ to $170{ }^{\circ} \mathrm{C}$ ). The decrease in resistance of the device indicates the semiconducting nature of sensing film and the trend may be attributed to increased charge carrier mobility at higher temperature. The resistance variation has been measured to be 1.17 times in the temperature range of $170{ }^{\circ} \mathrm{C}$ to $30^{\circ} \mathrm{C}$. In sealed environment, it is assumed that sensor design may be suitable for elevated temperature applications as well. Further, the environmental temperature normally does not vary much, so we propose, for the practical application of our fabricated humidity sensor, that the temperature compensation may not be required.

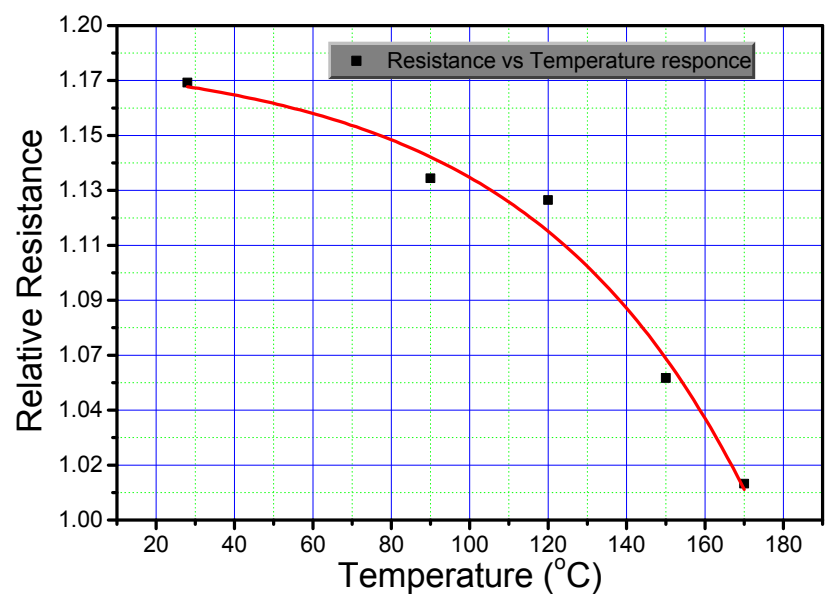

Figure 5. Resistance and temperature interdependence of organic-inorganic hybrid composite film. 


\section{Conclusions}

The interdependence of the humidity levels and electrical parameters of the sensor was studied in a wide frequency range $(300 \mathrm{~Hz}-100 \mathrm{KHz})$. Frequency study enabled better comprehension of the working mechanism of the humidity sensor under investigation. Capacitance was found to increase and the real component of impedance was found to decrease with the humidity level increment. Further the capacitance and impedance variation with humidity have been found to be strongly dependent upon input signal's frequency. The sensors showed high humidity sensing capability at low frequencies. However, the variation in electrical parameters of the sensor with humidity was depressed at higher order of frequency which is attributed to low mobility of charge carriers. The measurement results also revealed that the sensor with interdigitated electrode configuration gives higher dielectric permittivity variation as compared to the rectangular one. Temperature dependence of the sensor's resistance was found to be almost negligible, so temperature compensation is not required in the impedance spectra of the humidity sensor.

Acknowledgments: Author is thankful to faculty of Engineering Sciences, Ghulam Ishaq Khan Institute of Engineering Sciences and Technology, Pakistan, for technical support to complete experiments. The authors would like to thank Dr. K. S. Karimov Faculty of Electrical and Electronics Engineering, Ghulam Ishaq Khan Institute of Engineering Sciences and Technology, Pakistan and Dr. Qayyum Zafar, University of Malaya, Kuala Lumpur, Malaysia, for their valuable comments and sharing thei knowledge in completing the analysis.

Conflicts of Interest: The author declares no conflict of interest.

\section{References}

1. Ruiz-Garcia, L.; Lunadei, L.; Barreiro, P.; Robla, I. A review of wireless sensor technologies and applications in agriculture and food industry: state of the art and current trends. Sensors 2009, 9, 4728-4750. [CrossRef] [PubMed]

2. Laville, C.; Pellet, C. Comparison of three humidity sensors for a pulmonary function diagnosis microsystem. Sens. J. IEEE 2002, 2, 96-101. [CrossRef]

3. Ahmad, Z.; Shuhail, M.; Ibrahim, I.; Al-Rawi, W.K.; Sulaiman, K.; Zafar, Q.; Sazal, A.H.; Shaameri, Z. MEH-PPV $/ \mathrm{Alq}_{3}$ donor-acceptor blend as a photodetector. Chin. Phys. B 2013, 22, 100701-100705. [CrossRef]

4. Karimov, K.; Moiz, S.; Tahir, M.M.; Ahmed, N.; Tariq, R.; Abbas, S.; Zafar, Q. Nickel phthalocynanine-metal Schottky diode as photodetector. J. Optoelectron. Adv. Mater. 2014, 16, 1430-1435.

5. Bouattoura, D.; Villon, P.; Farges, G. Dynamic programming approach for newborn's incubator humidity control. IEEE Trans. Biomed. Eng. 1998, 45, 48-55. [CrossRef] [PubMed]

6. Saleem, M.; Karimov, K.S.; Karieva, Z.; Mateen, A. Humidity sensing properties of CNT-OD-VETP nanocomposite films. Phys. E 2010, 43, 28-32. [CrossRef]

7. Chani, M.T.S.; Karimov, K.S.; Ahmad Khalid, F.; Raza, K.; Umer Farooq, M.; Zafar, Q. Humidity sensors based on aluminium phthalocyanine chloride thin films. Phys. E 2012, 45, 77-81. [CrossRef]

8. Kim, S.-J.; Park, J.-Y.; Lee, S.-H.; Yi, S.-H. Humidity sensors using porous silicon layer with mesa structure. J. Phys. D Appl. Phys. 2000, 33, 1781-1784. [CrossRef]

9. Mulla, I.; Chaudhary, V.; Vijayamohanan, K. Humidity sensing properties of boron phosphate. Sens. Actuators A 1998, 69, 72-76. [CrossRef]

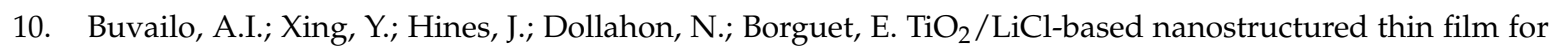
humidity sensor applications. ACS Appl. Mater. Interfaces 2011, 3, 528-533. [CrossRef] [PubMed]

11. Wang, C.-T.; Wu, C.-L.; Chen, I.; Huang, Y.-H. Humidity sensors based on silica nanoparticle aerogel thin films. Sens. Actuators B 2005, 107, 402-410. [CrossRef]

12. Innocenzi, P.; Zub, I.L.; Kessler, V.G. Sol-gel Methods for Materials Processing: Focusing on Materials for Pollution Control, Water Purification, and Soil Remediation; Springer: Dordrecht, Netherlands, 2008.

13. Ahmad, Z.; Zafar, Q.; Touati, F.; Shakoor, R.; Al-Thani, N. Study of $\pi$-conjugation effect of organic semiconductors on their optical parameters. Opt. Mater. 2016, 54, 94-97. [CrossRef]

14. Zafar, Q.; Aziz, F.; Sulaiman, K. Eco-benign visible wavelength photodetector based on phthalocyanine-low bandgap copolymer composite blend. RSC Adv. 2016, 6, 13101-13109. [CrossRef] 
15. Zafar, Q.; Ahmad, Z.; Sulaiman, K. PFO-DBT: MEH-PPV: PC71BM ternary blend assisted platform as a photodetector. Sensors 2015, 15, 965-978. [CrossRef] [PubMed]

16. Azmer, M.I.; Zafar, Q.; Ahmad, Z.; Sulaiman, K.; Karimov, K.S. VOPcPhO based organic pressure sensor and displacement transducer. Synth. Met. 2014, 191, 120-125. [CrossRef]

17. Zafar, Q.; Najeeb, M.A.; Ahmad, Z.; Sulaiman, K. Organic-inorganic hybrid nanocomposite for enhanced photo-sensing of PFO-DBT: MEH-PPV: PC71BM blend-based photodetector. J. Nanopart. Res. 2015, 17, 1-10. [CrossRef]

18. Ahmad, Z.; Abdullah, S.M.; Zafar, Q.; Sulaiman, K. Investigation of charge transport in organic polymer donor/acceptor photovoltaic materials. J. Mod. Opt. 2014, 61, 1730-1734. [CrossRef]

19. Azmer, M.I.; Zafar, Q.; Ahmad, Z.; Sulaiman, K. Humidity sensor based on electrospun MEH-PPV: PVP microstructured composite. RSC Adv. 2016, 6, 35387-35393. [CrossRef]

20. Zafar, Q.; Ahmad, Z.; Sulaiman, K.; Hamzah, A.S.; Rahman, Z.A. A MEHPPV/VOPcPhO composite based diode as a photodetector. Sens. Actuators A 2014, 206, 138-143. [CrossRef]

21. Ahmad, Z.; Sayyad, M.H.; Karimov, K.S. Bi-layer capacitive type light and humidity sensors. J. Ovonic Res. 2008, 5, 91-95.

22. Ahmad, Z.; Sayyad, M.H.; Karimov, K.S. Capacitive hygrometers based on natural organic compound. J. Optoelectron. Adv. Mater. 2008, 2, 507-510.

23. Ahmad, Z.; Sayyad, M.H.; Saleem, M.; Karimov, K.S.; Shah, M. Humidity-dependent characteristics of methyl-red thin film-based Ag/methyl-red/Ag surface-type cell. Phys. E Low-Dimens. Syst. Nanostruct. 2008, 41, 18-22. [CrossRef]

24. Ahmad, Z.; Sayyad, M.H.; Yaseen, M.; Aw, K.C.; M-Tahir, M.; Ali, M. Potential of 5,10,15,20-Tetrakis( $3^{\prime}, 5^{\prime}-$ di-tertbutylphenyl)porphyrinatocopper(II) for a multifunctional sensor. Sens. Actuators B 2011, 155, 81-85. [CrossRef]

25. Murtaza, I.; Karimov, K.S.; Ahmad, Z.; Qazi, I.; Mahroof-Tahir, M.; Khan, T.; Amin, T. Humidity sensitive organic field effect transistor. J. Semicond. 2010, 31. [CrossRef]

26. Aziz, F.; Hassan Sayyad, M.; Sulaiman, K.; Majlis, B.; Karimov, K.S.; Ahmad, Z.; Sugandi, G. IOPscienceInfluence of humidity conditions on the capacitive and resistive response of an $\mathrm{Al} / \mathrm{VOPc} / \mathrm{Pt}$ co-planar humidity sensor. Meas. Sci. Technol. 2012, 23. [CrossRef]

27. Aziz, F.; Sayyad, M.; Karimov, K.S.; Saleem, M.; Ahmad, Z.; Khan, S.M. Characterization of vanadyl phthalocyanine based surface-type capacitive humidity sensors. J. Semicond. 2010, 31. [CrossRef]

28. Shah, M.; Ahmad, Z.; Sulaiman, K.; Karimov, K.S.; Sayyad, M. Carbon nanotubes' nanocomposite in humidity sensors. Solid-State Electron. 2012, 69, 18-21. [CrossRef]

29. Shamala, K.; Murthy, L.; Radhakrishna, M.; Rao, K.N. Characterization of $\mathrm{Al}_{2} \mathrm{O}_{3}$ thin films prepared by spray pyrolysis method for humidity sensor. Sens. Actuators A 2007, 135, 552-557. [CrossRef]

30. Parvatikar, N.; Jain, S.; Khasim, S.; Revansiddappa, M.; Bhoraskar, S.; Prasad, M. Electrical and humidity sensing properties of polyaniline $/ \mathrm{WO}_{3}$ composites. Sens. Actuators B 2006, 114, 599-603. [CrossRef]

31. Suri, K.; Annapoorni, S.; Sarkar, A.; Tandon, R. Gas and humidity sensors based on iron oxide-polypyrrole nanocomposites. Sens. Actuators B 2002, 81, 277-282. [CrossRef]

32. Karimov, K.S.; Saleem, M.; Karieva, Z.; Mateen, A.; Chani, M.T.S.; Zafar, Q. Humidity sensing properties of Cu2O-PEPC nanocomposite films. J. Semicond. 2012, 33. [CrossRef]

33. Ahmad, Z.; Zafar, Q.; Sulaiman, K.; Akram, R.; Karimov, K.S. A humidity sensing organic-inorganic composite for environmental monitoring. Sensors 2013, 13, 3615-3624. [CrossRef] [PubMed]

34. Al-Sehemi, A.G.; Al-Assiri, M.S.; Kalam, A.; Zafar, Q.; Azmer, M.I.; Sulaiman, K.; Ahmad, Z. Sensing performance optimization by tuning surface morphology of organic (D- $\pi$-A) dye based humidity sensor. Sens. Actuators B 2016, 231, 30-37. [CrossRef]

35. Chani, M.T.S.; Karimov, K.S.; Khalid, F.A.; Moiz, S.A. Polyaniline based impedance humidity sensors. Solid State Sci. 2013, 18, 78-82. [CrossRef]

36. Ahmad, Z.; Abdullah, S.M.; Sulaiman, K. Temperature-sensitive chemical cell based on Nickel (II) phthalocyanine-tetrasulfonic acid tetrasodium salt. Sens. Actuators A 2012, 179, 146-150. [CrossRef] 
37. Gabriel, G.; Illa, X.; Guimera, A.; Rebollo, B.; Hernández-Ferrer, J.; Martin-Fernandez, I.; Martínez, M.T.; Godignon, P.; Sanchez-Vives, M.V.; Villa, R. Carbon Nanotubes as Suitable Interface for Improving Neural Recordings; INTECH Open Access Publisher: Rijeka, Croatia - EUROPEAN UNION, 2013.

38. Lin, Q.; Li, Y.; Yang, M. Investigations on the sensing mechanism of humidity sensors based on electrospun polymer nanofibers. Sens. Actuators B 2012, 171, 309-314. [CrossRef]

(C) 2016 by the author; licensee MDPI, Basel, Switzerland. This article is an open access article distributed under the terms and conditions of the Creative Commons Attribution (CC-BY) license (http:/ / creativecommons.org/licenses/by/4.0/). 